\title{
Eye Movements During Mindless Reading
}

Psychological Science

21 (9) $1300-1310$

(c) The Author(s) 2010

Reprints and permission:

sagepub.com/journalsPermissions.nav DOI: 10.1 1 177/0956797610378686

http://pss.sagepub.com

(S)SAGE

\author{
Erik D. Reichle', Andrew E. Reineberg', and Jonathan W. Schooler ${ }^{2}$ \\ 'University of Pittsburgh and ${ }^{2}$ University of California, Santa Barbara
}

\begin{abstract}
Mindless reading occurs when the eyes continue moving across the page even though the mind is thinking about something unrelated to the text. Despite how commonly it occurs, very little is known about mindless reading. The present experiment examined eye movements during mindless reading. Comparisons of fixation-duration measures collected during intervals of normal reading and intervals of mindless reading indicate that fixations during the latter were longer and less affected by lexical and linguistic variables than fixations during the former. Also, eye movements immediately preceding self-caught mind wandering were especially erratic. These results suggest that the cognitive processes that guide eye movements during normal reading are not engaged during mindless reading. We discuss the implications of these findings for theories of eye movement control in reading, for the distinction between experiential awareness and meta-awareness, and for reading comprehension.
\end{abstract}

\section{Keywords}

attention, awareness, eye movements, meta-awareness, reading

Received 6/9/09; Revision accepted I/7//0

Most readers have probably had the experience of moving their eyes across the text while at the same time their mind wandered so that nothing was comprehended. ... This "daydream mode" would be difficult to study experimentally (Rayner \& Fischer, 1996, p. 746).

The phenomenon of mindless reading is common - many people have had the experience of suddenly realizing that, although their eyes have been moving across the printed page, little or none of what they have been "reading" has been processed in a meaningful manner. Despite how frequently it occurs, however, very little is known about what happens in the mind during mindless reading. This is unfortunate, because, if estimates of how often the mind wanders are accurate (e.g., $30 \%$ of daily life; Kane et al., 2007), and if claims that mind wandering is detrimental to reading comprehension are correct (Schooler, McSpadden, Reichle, \& Smallwood, 2010; Schooler, Reichle, \& Halpern, 2004; Smallwood, McSpadden, $\&$ Schooler, 2008), then an understanding of mindless reading could prove highly beneficial. For example, being able to identify, in real time, when mindless reading is occurring might ameliorate a significant source of reading difficulty. Furthermore, the phenomenon of mindless reading provides an excellent contrast to normal reading by providing a window through which researchers can examine a variety of theoretical issues related to the perceptual and cognitive processes that support reading (Reichle, Liversedge, Pollatsek, \& Rayner,
2009). Examining mindless reading also allows researchers to investigate the nature of consciousness (Schooler, 2002).

The present study provides important new information about mindless reading by using an experience-sampling method that we used previously to study mind wandering in other tasks (including self-paced reading; Sayette, Reichle, \& Schooler, 2009). In the present study, we also used eyetracking technology to examine the moment-to-moment consequences of mind wandering during reading. One obvious advantage of using eye tracking is that it speaks directly to the nature of the eye-mind link - that is, the degree to which cognition plays an active role in guiding the eyes during reading (Rayner, 1998). Eye tracking also provides an extremely sensitive measure with which to ascertain the time course of mind wandering during reading - how often it occurs, how often readers vacillate between normal reading and mindless reading, and so on.

Theories of eye movement control during reading can be divided into two camps (for a review, see Reichle, Rayner, \& Pollatsek, 2003; Rayner, 2009). On one hand, cognitive-control theories posit a tight eye-mind link, with ongoing cognition (e.g., lexical processing) determining or modulating when the eyes will move from one word to the next (Engbert,

Corresponding Author:

Erik D. Reichle, 635 LRDC, University of Pittsburgh, Pittsburgh, PA I5260

E-mail: reichle@pitt.edu 
Nuthmann, Richter, \& Kliegl, 2005; Just \& Carpenter, 1980; Reichle, Pollatsek, Fisher, \& Rayner, 1998; Reilly \& Radach, 2006; Salvucci, 2001). On the other hand, oculomotor-control theories do not posit an eye-mind link, but instead maintain that readers' eye movement behavior is determined by global constraints imposed by visual and oculomotor factors (e.g., limited retinal acuity; Feng, 2006; McDonald, Carpenter, \& Shillcock, 2005; Yang, 2006). It is perhaps not too surprising that both classes of theory explain a wide range of phenomena associated with readers' eye movements, with theorists' attempts to use empirical data to adjudicate between the theories often producing equivocal results (cf. Inhoff, Eiter, \& Radach, 2005; Pollatsek, Reichle, \& Rayner, 2006).

One example that is particularly relevant to the experiment reported in this article involves a paradigm known as $z$-string reading (Nuthmann \& Engbert, 2009; Rayner \& Fischer, 1996; Vitu, O'Regan, Inhoff, \& Topolski, 1995). In this paradigm, participants are given strings of text comprising entirely the letter $z$ (e.g., "Zzz zzzzzz zz"). The participants are instructed to pretend that they are reading the $z$ strings while their eye movements are recorded. Some researchers might argue that, to the degree that eye movements during $z$ reading resemble eye movements during normal reading, cognition (e.g., lexical access) plays little or no role, and that eye movements are instead guided by visual or oculomotor factors. Although fixations tend to be longer in $z$ reading than in normal reading, and although the effects of lexical variables (e.g., word frequency) are necessarily absent in $z$ reading, the experiments have been inconclusive: Advocates of oculomotor-control theories claim that eye movements during $z$ reading resemble eye movements during normal reading (Vitu et al., 1995), and advocates of cognitive-control theories deny this claim (Rayner \& Fischer, 1996). The experiments are also inherently difficult to evaluate because it is not clear what participants are actually doing when they are pretending to read $z$ strings.

The present experiment avoided the limitations of the $z$-reading paradigm by measuring readers' eye movements during periods of both normal reading and actual mindless reading. The logic of this approach was that, by comparing the variables that influence eye movements in normal reading and mindless reading, we would be able to assess the degree to which cognition influences eye movements during normal reading. Our central hypothesis was that fixations during mindless reading should be qualitatively different than fixations associated with normal reading; specifically, fixations during mindless reading should have longer durations (as observed during $z$ reading) and less sensitivity to lexical variables (e.g., word frequency) than fixations during normal reading. These effects are normally indicative of on-line cognitive processing.

The present study also explored the potential role of metaawareness (Schooler, 2002) in modulating such differences. Simply put, meta-awareness refers to intermittent periods when people become aware of their awareness, to explicitly take stock of whatever they are thinking about. In contrast, experiential awareness corresponds to what people normally experience when engaged in any ongoing activity or task- the normal stream of consciousness. In the present study, experiential awareness corresponds to what transpires during normal reading when a person is engaged in the text, as well as during mindless reading when a person is engaged in thoughts unrelated to the text. Meta-awareness, then, occurs when a person realizes that his or her mind has been wandering.

Prior research (Schooler et al., 2004) has used two measures to distinguish mind-wandering episodes that occur with awareness versus without awareness: (a) Self-caught mind wandering, in which participants press a button whenever they notice their mind wandering, indicates episodes that individuals have become self-aware of, and (b) probe-caught mind wandering, in which participants are caught mind wandering by random experience-sampling probes, indicates episodes that have (until the probe) evaded meta-awareness. Differences between self-caught and probe-caught measures have helped illuminate the role of meta-awareness in mind wandering. For example, alcohol consumption (Sayette et al., 2009) attenuates meta-awareness of mind wandering; this attenuation is evidenced by dramatic reductions in the ratio of selfcaught to probe-caught episodes. Given the differential sensitivity of these two measures to meta-awareness, we predicted differences in eye movements prior to self-caught and probe-caught mind wandering. Our assumption was that the former should be associated with participants' dawning awareness of having lapsed into mindless reading.

\section{Method \\ Participants}

Four undergraduates ( 3 females and 1 male) at the University of Pittsburgh participated for payment. Participants were paid $\$ 7$ per hour, with a $\$ 20$ bonus for completing the experiment. All subjects were native English speakers with normal vision. None of the participants were familiar with the text used in the study.

\section{Apparatus}

An EyeLink 1000 eye tracker (SR Research, Ottawa, Ontario, Canada) monitored the gaze location of participants' right eye during reading. The eye tracker had a spatial resolution of 30 arcmin and a $1000-\mathrm{Hz}$ sampling rate. Participants viewed the stimuli binocularly on a monitor $63 \mathrm{~cm}$ from their eyes; 3.1 characters of text equaled approximately $1^{\circ}$ of visual angle. Chin and forehead rests were used to minimize head movements and ensure comfort.

\section{Materials}

Participants read the entirety of Sense and Sensibility by Jane Austen (1811/2008). The novel consisted of 50 chapters, with 
7 to 17 pages per chapter and a maximum of 25 lines per page. Four-alternative multiple-choice questions were created (3-4 per chapter) to measure participants' comprehension of the material. Participants read the material over multiple days so they could adapt to both reading on the eye tracker and the general procedure; this was done to make the reading as routine as possible and thereby maximize the probability of observing mindless reading. ${ }^{1}$

\section{Procedure}

Each participant read the novel at his or her own pace across 12 to $15(M=13.5)$ hour-long sessions. Each session and chapter began with a calibration of the eye tracker. Calibration was not done more frequently to avoid being intrusive and possibly reducing the frequency of mind wandering. Participants read at their own pace by pressing the "F" key to move forward through the text and pressing the "B" key to move backward through the text. Participants were provided with the following definition of zoning out: "At some point during reading, you realize that you have no idea what you just read" and that "not only were you not thinking about the text, you were thinking about something else altogether." Participants were instructed to press the "Z" key whenever they caught themselves zoning out. Participants were also prompted every 2 to 4 min (time sampled randomly from a uniform distribution) after the previous self-reported zone out or prompt to indicate whether they had been zoning out at the time of the prompt; participants were instructed to press the "Y" key if they had and the "N" key if they had not. During the last $5 \mathrm{~min}$ of each session, participants answered comprehension questions about the chapters they had read.

\section{Results}

\section{Behavioral measures}

Table 1 shows several behavioral measures for each participant, including the total number of hour-long sessions that the participant spent reading, the participant's overall accuracy in answering text-related comprehension questions, the number of times the participant caught him- or herself zoning out (self-caught zone outs), and the number of probes that caught the participant zoning out (probe-caught zone outs). To adjust for differences in the total number of probes participants received, we also report the probe-caught ratio, or the proportion of probes that caught participants zoning out.

On average, participants correctly answered $81 \%$ of the comprehension questions (chance $=25 \%$ ); this percentage indicates that they understood the text. Participants selfreported zoning out 8 to 36 times during the study $(M=22.5)$ and were caught zoning out by $4.5 \%$ to $15.3 \%$ of the probes $(M=9.3 \%)$. The mean result is particularly striking because it suggests that participants were zoning out approximately $9 \%$ of the time (on average) without being aware that they were doing so. All of these mind-wandering results are consistent with the results of previous studies, which have used selfpaced reading (but not eye tracking) to study mind wandering during reading (Sayette et al., 2009; Schooler et al., 2004).

\section{Global eye movement measures}

We assessed participants' eye movements during the 120 s preceding three conditions, which represented the points in time when participants (a) pressed a button to indicate self-caught zone outs (i.e., self-caught mindless reading), (b) responded affirmatively to probes that asked whether they had been zoning out (i.e., probe-caught mindless reading), and (c) responded negatively to probes that asked whether they had been zoning out (i.e., normal reading). Individual fixation locations and durations were extracted (using Pegasus software; Loboda, 2009) from the continuous coverage of participants' eye movements obtained by the eye tracker. Fixation data were taken from six intervals $(2.5 \mathrm{~s}, 5 \mathrm{~s}, 10 \mathrm{~s}, 30 \mathrm{~s}, 60 \mathrm{~s}$, and $120 \mathrm{~s})$ preceding each condition. The 120 -s interval thus spanned the minimal time between the preceding prompt or self-reported zone out and the current prompt (2-4 min).

Individual fixations that began prior to the interval onsets and extended into the measured interval range were excluded from analyses to equate for the overall interval lengths across conditions. The last fixation within each interval was also excluded because it was interrupted either by participants pressing the " $Z$ " button (in the self-caught condition) or by probes (in the other two conditions). Finally, although fixation

Table I. Behavioral Measures

\begin{tabular}{lcccccc}
\hline Participant & $\begin{array}{c}\text { Reading time } \\
\text { (hours) }\end{array}$ & $\begin{array}{c}\text { Comprehension } \\
\text { accuracy }\end{array}$ & $\begin{array}{c}\text { Number of self- } \\
\text { caught zone outs }\end{array}$ & $\begin{array}{c}\text { Number of } \\
\text { probes }\end{array}$ & $\begin{array}{c}\text { Number of probe- } \\
\text { caught zone outs }\end{array}$ & $\begin{array}{c}\text { Probe-caught } \\
\text { ratio }\end{array}$ \\
\hline $\mathrm{I}$ & 15 & .801 & 36 & 179 & 8 & .045 \\
2 & 12 & .754 & 25 & 124 & 19 & .153 \\
3 & 13 & .895 & 8 & 148 & 20 & .135 \\
4 & 14 & .807 & 21 & 155 & 6 & .039 \\
$M$ & 13.5 & .814 & 22.50 & 151.50 & 13.25 & .093 \\
SE & 0.65 & .029 & 5.78 & 11.32 & 3.64 & .030 \\
\hline
\end{tabular}

Note: The probe-caught ratio indicates the proportion of probes that caught participants zoning out. 
durations less than $80 \mathrm{~ms}$ or more than $1,000 \mathrm{~ms}$ are typically discarded as outliers in eye movement experiments (Inhoff \& Radach, 1998; Liversedge, Paterson, \& Pickering, 1998), this precaution was not followed in the present study because of the possible erratic nature of eye movements during mindless reading (e.g., fixations were predicted to be longer than normal).

Figure 1 shows several global eye movement measures for the six intervals. These measures include mean numbers for four variables: first-pass fixations (i.e., fixations on a word during the first pass through the text), interword regressions (i.e., saccades back to words that occurred earlier in the text), words fixated (i.e., gazes on individuals words), and off-text fixations (e.g., gazes not on individual words). Data from each interval were examined using repeated measures analyses of variance (ANOVAs) with one factor (normal reading vs. selfcaught mindless reading vs. probe-caught mindless reading). ANOVAs indicating significant or marginally significant results (all $F_{\mathrm{s}} \geq 4.20$, all $p \mathrm{~s} \leq .072$ ) were further examined using two-tailed matched-sample $t$ tests. These contrasts indicated that there were fewer first-pass fixations, $t(3)=6.88, p=$ .006 , Cohen's $d=7.94$; fewer interword regressions, $t(3)=$ 9.32, $p=.003, d=10.76$; and fewer words fixated, $t(3)=5.40$, $p=.012, d=6.24$; but more off-text fixations, $t(3)=4.72, p=$ $.018, d=5.45$, during the $2.5 \mathrm{~s}$ immediately preceding selfcaught zone outs than during normal reading. There were also fewer first-pass fixations, $t(3)=7.14, p=.006, d=8.24$, and words fixated, $t(3)=8.20, p=.004, d=9.47$, during the 2.5 -s interval preceding self-caught zone outs than during the 2.5 -s interval preceding probe-caught zone outs.

These differences suggest that participants' awareness of mind wandering immediately prior to self-reporting zone outs may manifest itself in more erratic patterns of eye movements than the eye movements either during normal reading or prior to probe-caught zone outs. There were also more off-text fixations during probe-caught mindless reading than during both normal reading (both $t \mathrm{~s} \geq 3.72$, both $p \mathrm{~s} \leq .034$, both $d \mathrm{~s} \geq 4.30$ ) and self-caught mindless reading, $t(3)=4.58, p=.019, d=5.29$,

\section{Normal Reading $\square$ Self-Caught Mindless Reading $\square$ Probe-Caught Mindless Reading}

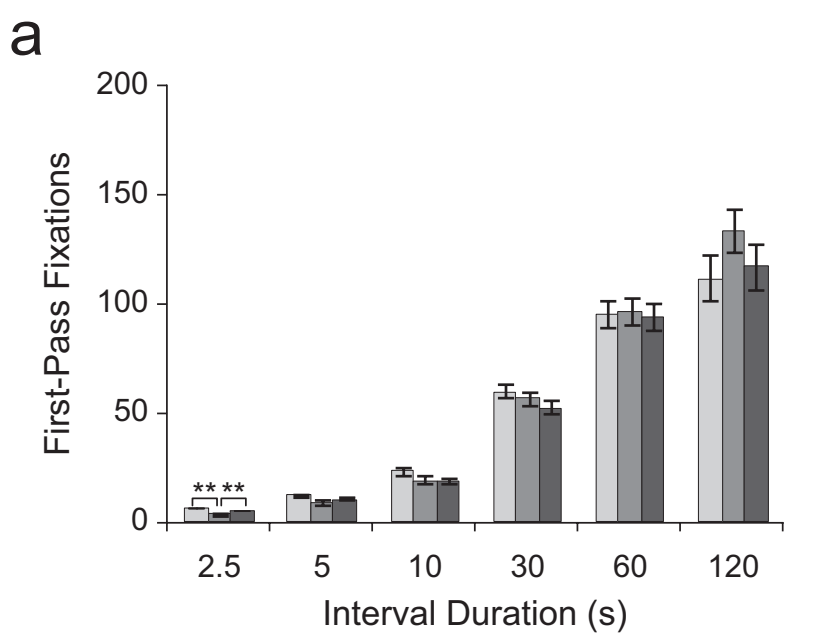

b
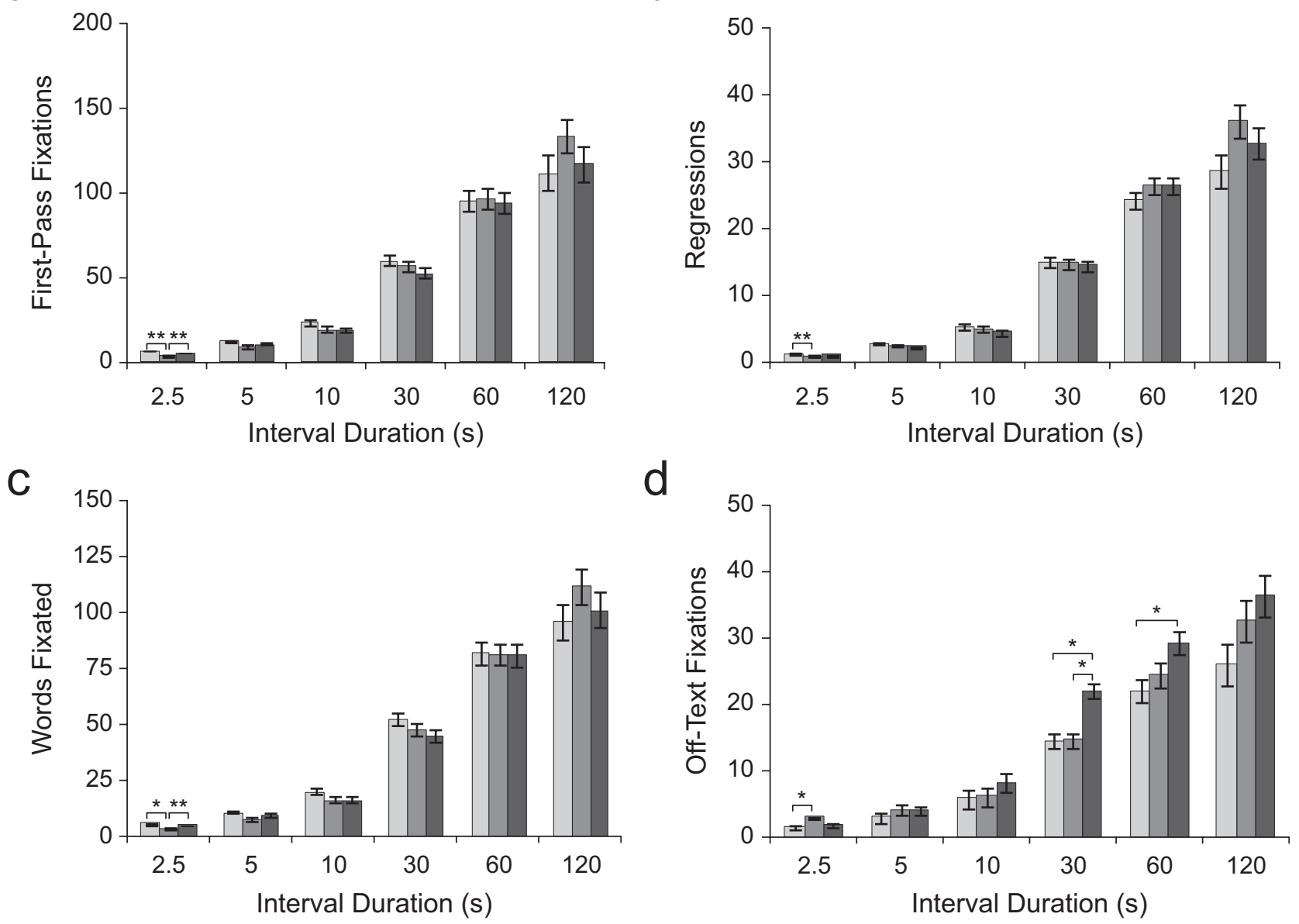

Fig. I. Mean global eye movement measures during normal reading, self-caught mindless reading, and probe-caught mindless reading as a function of interval length. Mean numbers were calculated for (a) first-pass fixations, (b) interword regressions, (c) words fixated, and (d) off-text fixations. The error bars indicate standard errors of the mean, with significant differences indicated $\left({ }^{*} p<.05\right.$, $\left.{ }^{*} p<.0 \mathrm{I}\right)$. 
in two of the longer intervals; however, these erratic eye movements were not associated with participants' self-awareness of mind wandering because participants did not catch themselves mind wandering but were instead caught by probes.

\section{Local eye movement measures}

Figure 2 shows three local measures of eye movement behavior: first-fixation duration, or the duration of the initial fixation on a word during the first pass through the text; gaze duration, or the sum of all first-pass fixations on a word; and total viewing time, or the sum of all fixations on a word (including those occurring after interword regressions). These measures are reported for fixations taken from the same intervals used in the previous analyses. These measures also reflect the full time course of processing: Whereas first-fixation duration and gaze duration are early measures that are influenced by a variety of lower-level lexical variables (e.g., word frequency; Rayner \& Duffy, 1986; Schilling, Rayner, \& Chumbley, 1998), total viewing time is a late measure influenced by higher-level linguistic variables (e.g., semantic plausibility; Clifton, Staub, \& Rayner, 2007).

As with the first set of analyses, data from each interval were examined using repeated measures ANOVAs, with all significant or marginally significant results (all $F \mathrm{~s} \geq 3.49$, all $p \mathrm{~s} \leq .099$ ) then being examined using one-tailed $t$ tests (for normal reading vs. mindless reading) and two-tailed $t$ tests (for self-caught mindless reading vs. probe-caught mindless reading). These contrasts indicated that first-fixation durations, gaze durations, and total viewing times were shorter for normal reading than for self-caught mindless reading during the four longest intervals (all $t \mathrm{~s} \geq 2.35$, all $p \mathrm{~s} \leq .05$, all $d \mathrm{~s} \geq 2.71$ ).

Gaze durations were also shorter for normal reading than for probe-caught mindless reading during the 30 -s intervals, $t(3)=4.34, p=.012, d=5.01$, and 120-s intervals, $t(3)=2.43$, $p=.047, d=2.81$, and total viewing times were shorter for normal reading than for probe-caught mindless reading during the 30-s intervals, $t(3)=4.12, p=.013, d=4.76$, and 60-s intervals, $t(3)=2.59, p=.041, d=2.99$. As Figure 2 shows, however, the fixation-duration measures tended to be shorter during probe-caught than self-caught mindless reading, particularly for total viewing times during the 10-s intervals, $t(3)=$ $3.88, p=.03, d=4.48$. These results indicate that longer fixations are indicative of mindless reading as opposed to normal reading, and that the mind appears to wander for a considerable amount of time (up to $120 \mathrm{~s}$ ) before being caught; however, these behaviors appear to be more pronounced in self-caught mindless reading. This suggests that the lengthening of fixations may be one factor that contributed to participants' awareness of having lapsed into mind wandering (although see the General Discussion for an alternative account).

To determine whether lexical or linguistic processing affected eye movements during mindless reading, we completed
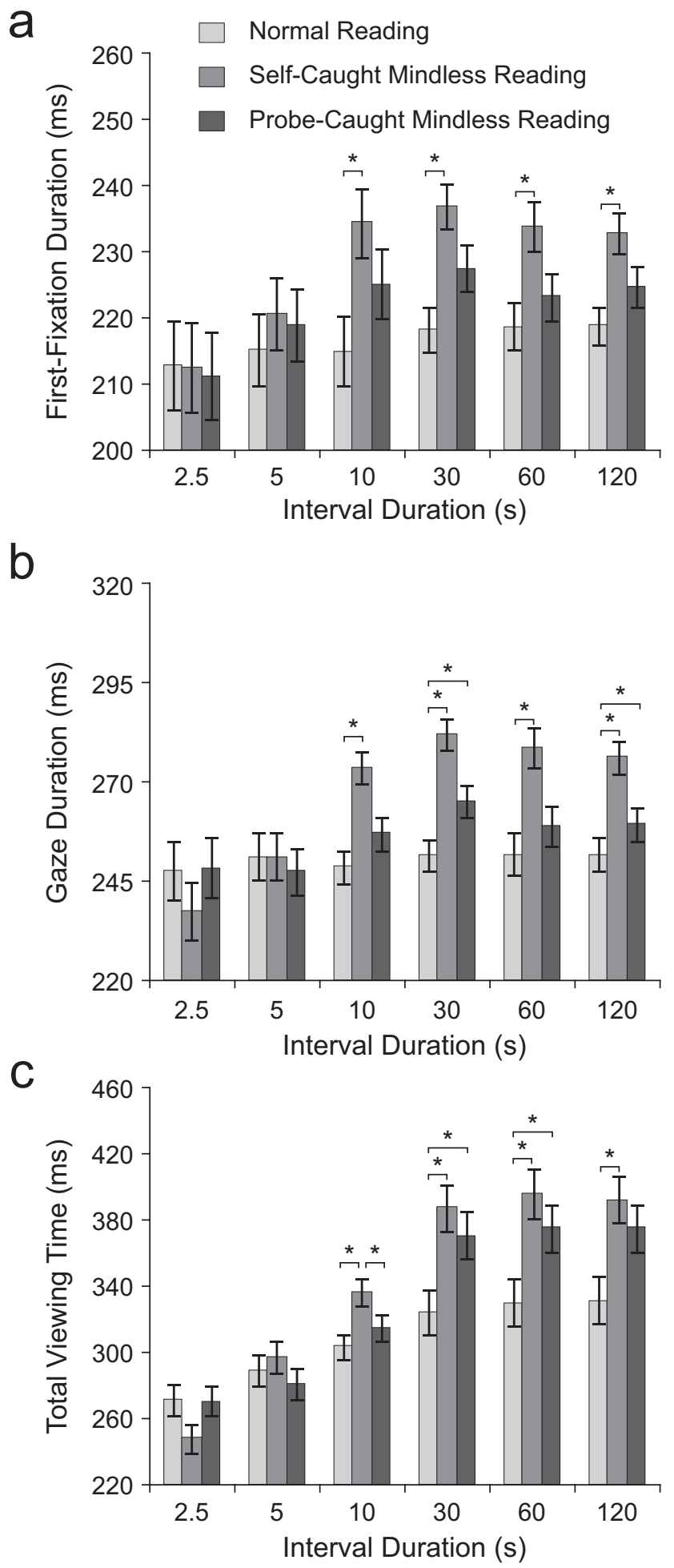

Fig. 2. Mean local eye movement measures during normal reading, selfcaught mindless reading, and probe-caught mindless reading as a function of interval length. Means were calculated for (a) first-fixation durations, (b) gaze durations, and (c) total viewing times. The error bars indicate standard errors of the mean, with significant differences indicated $(* p<.05)$.

several stepwise multiple regression analyses (one for each interval) to control for between-participants differences. We used three variables as predictors of first-fixation duration, 
gaze duration, and total viewing time: the inverse of word length, the natural logarithm of word frequency (as tabulated by Francis \& Kucera, 1982), and whether the words occurred at the ends of clauses or sentences. Tables 2 through 4 show the results of these analyses, which indicated that all three predictor variables had more pronounced effects on the fixationduration measures during normal reading than during mindless reading.

The finding that fixation durations were shorter during normal reading for shorter words and for more frequent words is consistent with numerous eye movement experiments (Just \& Carpenter, 1980; Kliegl, Grabner, Rolfs, \& Engbert, 2004) and may reflect some combination of both sublexical processing (e.g., the extraction of orthographic information) and lexical processing (e.g., the activation of word meaning). It is unclear why the clause-/sentence-final words were fixated on for less time during normal reading than during mindless reading (clause-/sentence-final words are usually the recipients of longer fixations; Just \& Carpenter, 1980; Rayner, Sereno, Morris, Schmauder, \& Clifton, 1989; Warren, White, \& Reichle, 2009). One possible explanation for this discrepancy is that the semantic and syntactic content of the novel used in the present study was somewhat redundant, making clause or sentence "wrap up" less necessary or easier.

The key finding that fixation-duration measures were less affected by predictor variables during mindless reading suggests that decisions about when to move the eyes are less affected by cognitive processing during mindless reading than during normal reading, but that this reduction of cognitive control (on average) becomes more pronounced over time. Because of the inherent variability of eye movement data, however, it is not possible to know whether our participants mind-wandered until they were caught, or whether they alternated between brief periods of mindless reading and mindful reading. It is therefore impossible to know whether lexical and linguistic processing completely stops during mind wandering, or whether processing intermittently stops and starts. It is also possible that the two types of processing are differentially engaged during mindless reading (e.g., lexical processing might be more likely to start and stop but linguistic processing might be more likely to completely stop). Future research is clearly needed to examine this important issue.

Finally, because our method is inherently correlational, it was important to determine whether properties of the text

Table 2. Significant Results of Multiple Regression Analyses of Dependent Measures During Normal Reading

\begin{tabular}{|c|c|c|c|c|}
\hline \multirow[b]{2}{*}{$\begin{array}{l}\text { Interval and } \\
\text { dependent measure }\end{array}$} & \multirow[b]{2}{*}{ Constant $\left(b_{0}\right)$} & \multicolumn{3}{|c|}{ Predictor variable } \\
\hline & & $\begin{array}{c}\text { Inverse of } \\
\text { word length }\left(b_{1}\right)\end{array}$ & $\begin{array}{l}\text { Natural logarithm of } \\
\text { word frequency }\left(b_{2}\right)\end{array}$ & $\begin{array}{l}\text { Clause-/sentence- } \\
\text { final word }\left(b_{3}\right)\end{array}$ \\
\hline \multicolumn{5}{|l|}{$2.5 \mathrm{~s}$} \\
\hline First-fixation duration & 211 & - & - & - \\
\hline Gaze duration & 287 & $-5.99 *$ & $-2.04 *$ & - \\
\hline Total viewing time & 322 & $-70.11 * *$ & $-3.13^{* *}$ & - \\
\hline \multicolumn{5}{|l|}{$5 s$} \\
\hline First-fixation duration & 202 & - & $0.93 *$ & - \\
\hline Gaze duration & 296 & $-59.89 * * *$ & $-2.21 * *$ & - \\
\hline Total viewing time & 349 & $-83.87 * * * *$ & $-4.08 * * *$ & - \\
\hline \multicolumn{5}{|l|}{$10 \mathrm{~s}$} \\
\hline First-fixation duration & 204 & $23.13 * * *$ & - & $-7.75^{*}$ \\
\hline Gaze duration & 297 & $-42.66 * * * *$ & $-2.92 * * *$ & - \\
\hline Total viewing time & 385 & $-101.86^{* * *}$ & $-5.31 * * *$ & - \\
\hline \multicolumn{5}{|l|}{$30 \mathrm{~s}$} \\
\hline First-fixation duration & 211 & - & - & $-9.2 * * *$ \\
\hline Gaze duration & 309 & $-62.6 I^{* * * *}$ & $-3.26 * * * *$ & $-7.42^{*}$ \\
\hline Total viewing time & 442 & $-149.94 * * *$ & $-6.68 * * *$ & $-13.79 * *$ \\
\hline \multicolumn{5}{|l|}{$60 \mathrm{~s}$} \\
\hline First-fixation duration & 209 & - & $0.4 I^{*}$ & $-10.34 * * *$ \\
\hline Gaze duration & 308 & $-60.86 * * *$ & $-3.18 * * *$ & - \\
\hline Total viewing time & 462 & $-159.52 * * *$ & $-7.17 * * *$ & $-13.3 * * *$ \\
\hline \multicolumn{5}{|l|}{$120 \mathrm{~s}$} \\
\hline First-fixation duration & 213 & - & - & $-12 * * *$ \\
\hline Gaze duration & 317 & $-70.47 * * *$ & $-4 * * *$ & $-9.58 * * *$ \\
\hline Total viewing time & 490 & $-180.65 * * *$ & $-18.11 * * * *$ & $-22.5^{* * *}$ \\
\hline
\end{tabular}

$*_{t}(223 \mathrm{I}) \geq 1.96, p<.05 . * * t(223 \mathrm{I}) \geq 2.58, p<.01 . * * * t(223 \mathrm{I}) \geq 3.29, p<.001$. 
Table 3. Significant Results of Multiple Regression Analyses of Dependent Measures During Self-Caught Zone Outs

\begin{tabular}{|c|c|c|c|c|}
\hline \multirow[b]{2}{*}{$\begin{array}{l}\text { Interval and } \\
\text { dependent measure }\end{array}$} & \multirow[b]{2}{*}{ Constant $\left(b_{0}\right)$} & \multicolumn{3}{|c|}{ Predictor variable } \\
\hline & & $\begin{array}{c}\text { Inverse of } \\
\text { word length }\left(b_{1}\right)\end{array}$ & $\begin{array}{l}\text { Natural logarithm of } \\
\text { word frequency }\left(b_{2}\right)\end{array}$ & $\begin{array}{l}\text { Clause-/sentence- } \\
\text { final word }\left(b_{3}\right)\end{array}$ \\
\hline \multicolumn{5}{|l|}{$2.5 \mathrm{~s}$} \\
\hline First-fixation duration & 207 & - & - & $-37.17^{*}$ \\
\hline Gaze duration & 226 & - & - & - \\
\hline Total viewing time & 237 & - & - & - \\
\hline \multicolumn{5}{|l|}{$5 \mathrm{~s}$} \\
\hline First-fixation duration & 215 & - & - & - \\
\hline Gaze duration & 233 & - & - & - \\
\hline Total viewing time & 253 & - & - & - \\
\hline \multicolumn{5}{|l|}{$10 \mathrm{~s}$} \\
\hline First-fixation duration & 233 & - & - & - \\
\hline Gaze duration & 274 & - & - & - \\
\hline Total viewing time & 283 & $-151.69 * *$ & - & - \\
\hline \multicolumn{5}{|l|}{$30 \mathrm{~s}$} \\
\hline First-fixation duration & 223 & $33.47^{*}$ & - & $-14.85^{*}$ \\
\hline Gaze duration & 310 & - & $-3.93 * * *$ & - \\
\hline Total viewing time & 499 & $-136.02 * *$ & $-10.58 * * *$ & - \\
\hline \multicolumn{5}{|l|}{$60 \mathrm{~s}$} \\
\hline First-fixation duration & 216 & - & - & $-13.37 * *$ \\
\hline Gaze duration & 292 & $-87.22 * * *$ & - & - \\
\hline Total viewing time & 499 & $-188.94 * * *$ & $-6.66 * * *$ & - \\
\hline \multicolumn{5}{|l|}{$120 \mathrm{~s}$} \\
\hline First-fixation duration & 223 & $-17.35^{* * *}$ & - & - \\
\hline Gaze duration & 317 & $-111.2 * * *$ & - & $-15.65^{*}$ \\
\hline Total viewing time & 515 & $-239.3 * * *$ & $-6.68 * * *$ & $-24.86^{*}$ \\
\hline
\end{tabular}

$* t(307) \geq 1.96, p<.05 . * * t(307) \geq 2.58, p<.01 . * * * t(307) \geq 3.29, p<.001$.

(e.g., overall difficulty) contributed to the observed differences between normal reading and mindless reading. We addressed this issue in two ways. First, we completed ANOVAs and $t$ tests (as in our previous analyses) on three properties of the words included in our previous analyses: inverse word length, natural logarithm of word frequency, and the number of clause-/sentence-final words. As Figure 3 shows, these analyses revealed only four significant differences. Words were shorter during normal reading than during selfcaught mindless reading in the 10-s intervals, $t(3)=3.47, p=$ $.04, d=4.01$, and 60-s intervals, $t(3)=3.31, p=.045, d=$ 3.82 ; words were more frequent during normal reading than during self-caught mindless reading in the 60 -s intervals, $t(3)=3.75, p=.033, d=4.33$; and there were more clause-/ sentence-final words during probe-caught than during selfcaught mindless reading in the 5-s intervals, $t(3)=4.96, p=$ $.016, d=5.73$.

Second, we examined global measures of eye movement behavior, fixation-duration measures, and properties of words during a 120 -s interval following participants' responses to probes (after indicating normal reading and probe-caught mindless reading) and after self-reported zone outs. ${ }^{2}$ As Figure 4 shows, there was only one significant difference: Words were longer in self-caught mindless reading than in normal reading, $t(3)=4.04, p=.027, d=4.66$. Together, these analyses strongly suggest that the observed differences between mindless reading and normal reading were not driven by differences in the text being sampled, and that the patterns of eye movements indicative of mindless reading were specific to intervals of mindless reading.

\section{General Discussion}

The present study revealed several differences between the eye movements observed during normal reading and during mindless reading. The first difference is that fixation-duration measures were longer during self-caught and probe-caught mindless reading than during normal reading, and that these differences were evident as early as 60 s to 120 s prior to when the mind wandering was caught; however, this pattern was more pronounced for self-caught episodes. The second difference is that the fixation-duration measures were less affected 
Table 4. Significant Results of Multiple Regression Analyses of Dependent Measures During Probe-Caught Zone Outs

\begin{tabular}{|c|c|c|c|c|}
\hline \multirow[b]{2}{*}{$\begin{array}{l}\text { Interval and } \\
\text { dependent measure }\end{array}$} & \multirow[b]{2}{*}{ Constant $\left(b_{0}\right)$} & \multicolumn{3}{|c|}{ Predictor variable } \\
\hline & & $\begin{array}{c}\text { Inverse of } \\
\text { word length }\left(b_{1}\right)\end{array}$ & $\begin{array}{l}\text { Natural logarithm of } \\
\text { word frequency }\left(b_{2}\right)\end{array}$ & $\begin{array}{l}\text { Clause-/sentence- } \\
\text { final word }\left(b_{3}\right)\end{array}$ \\
\hline \multicolumn{5}{|l|}{$2.5 \mathrm{~s}$} \\
\hline First-fixation duration & 209 & - & - & - \\
\hline Gaze duration & 238 & - & - & - \\
\hline Total viewing time & 251 & - & - & - \\
\hline \multicolumn{5}{|l|}{$5 s$} \\
\hline First-fixation duration & 201 & - & - & - \\
\hline Gaze duration & 289 & $-111.92 * *$ & - & - \\
\hline Total viewing time & 323 & $-131.85^{* *}$ & - & - \\
\hline \multicolumn{5}{|l|}{$10 \mathrm{~s}$} \\
\hline First-fixation duration & 222 & - & - & - \\
\hline Gaze duration & 329 & $-78.39 *$ & $-3.95 *$ & - \\
\hline Total viewing time & 390 & $-219.21 * * *$ & - & - \\
\hline \multicolumn{5}{|l|}{$30 \mathrm{~s}$} \\
\hline First-fixation duration & 248 & - & - & - \\
\hline Gaze duration & 359 & $-108.28 * * *$ & $-4.01 * * * *$ & - \\
\hline Total viewing time & 500 & $-203.33 * * *$ & $-6.84 * * *$ & - \\
\hline \multicolumn{5}{|l|}{$60 \mathrm{~s}$} \\
\hline First-fixation duration & 244 & - & - & - \\
\hline Gaze duration & 368 & $-100.5^{* * *}$ & $-5.39 * * *$ & - \\
\hline Total viewing time & 544 & $-246.35^{* * *}$ & $-9.08 * * * *$ & - \\
\hline \multicolumn{5}{|l|}{$120 s$} \\
\hline First-fixation duration & 234 & - & - & $-7.42 *$ \\
\hline Gaze duration & 366 & $-111.27 * * *$ & $-5.21 * * *$ & - \\
\hline Total viewing time & 566 & $-288.48 * * *$ & $-9.49 * * *$ & - \\
\hline
\end{tabular}

$*_{t}(230) \geq 1.96, p<.05 . * * t(230) \geq 2.58, p<.01 . * * * t(230) \geq 3.29, p<.001$.

by ongoing lexical and linguistic processing during the 10 s to $30 \mathrm{~s}$ prior to mind wandering being caught than during the same intervals during normal reading. Finally, participants were less likely to make first-pass fixations, word fixations, or interword regressions (all of which are indicative of normal text processing) in the 2.5 -s interval immediately preceding self-caught mind wandering than in the 2.5 -s interval preceding either probe-caught mind wandering or normal reading; participants were instead more likely to be looking somewhere other than the text. We discuss the theoretical implications of these findings in turn.

The findings that fixations become longer in duration and progressively less sensitive to lexical and linguistic variables suggest that readers lapse into periods of mind wandering that are extensive in duration (1-2 $\mathrm{min}$ ) and that, during these lapses, eye movements become progressively decoupled from ongoing text processing. One implication of these results concerns the nature of eye movement control during normal reading: When participants were reading mindfully, their eye movements showed a sensitivity to lexical and linguistic variables that was less apparent when they were reading mindlessly.
This is consistent with the hypothesis that decisions about when to move the eyes are normally related to cognitive processing, as stipulated by cognitive-control theories (Engbert et al., 2005; Just \& Carpenter, 1980; Reichle et al., 1998; Reilly \& Radach, 2006; Salvucci, 2001).

These differences also further document the tractability of mindless reading as a topic of investigation (Sayette et al., 2009; Schooler et al., 2004, 2009; Smallwood et al., 2008) by providing the first demonstration that eye movements can be used as on-line indicators of mind wandering. This covert measure of mind wandering may also enable the investigation of questions that have been impossible to address with intrusive self-report measures. For example, is it possible to develop an on-line method to catch people mind wandering before they notice it themselves? If so, can comprehension be improved by sensitizing people to mindless reading? Although the resolution of such questions awaits future research, the present study indicates that eye tracking provides an invaluable tool for studying mindless reading, and that eye tracking may ultimately lead to technology to ameliorate a major source of comprehension difficulty. 
a

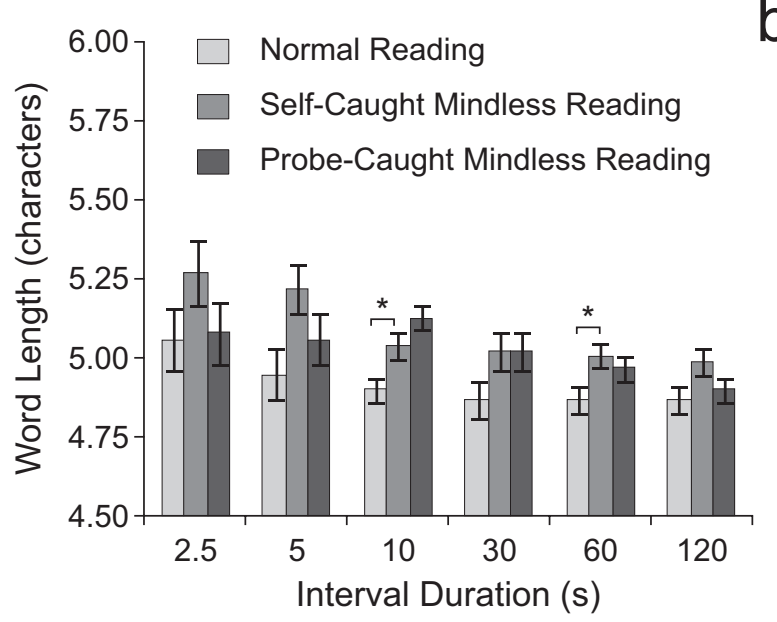

b

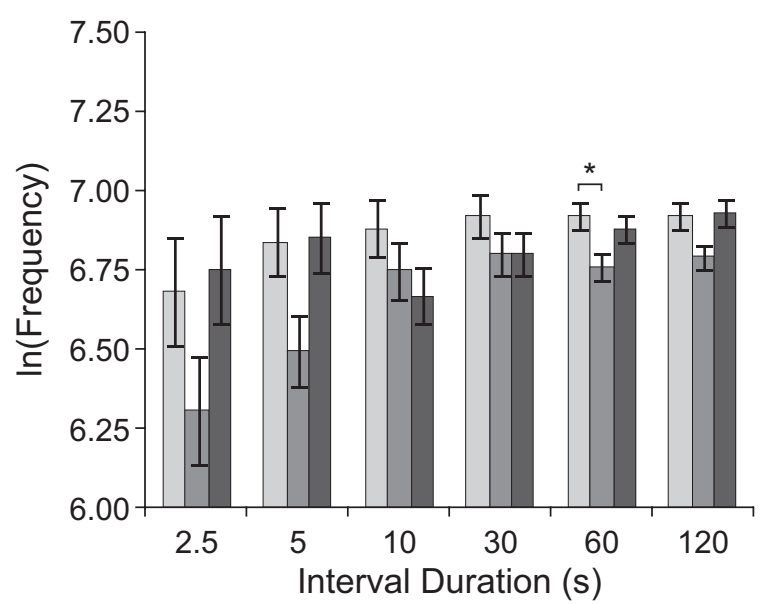

C

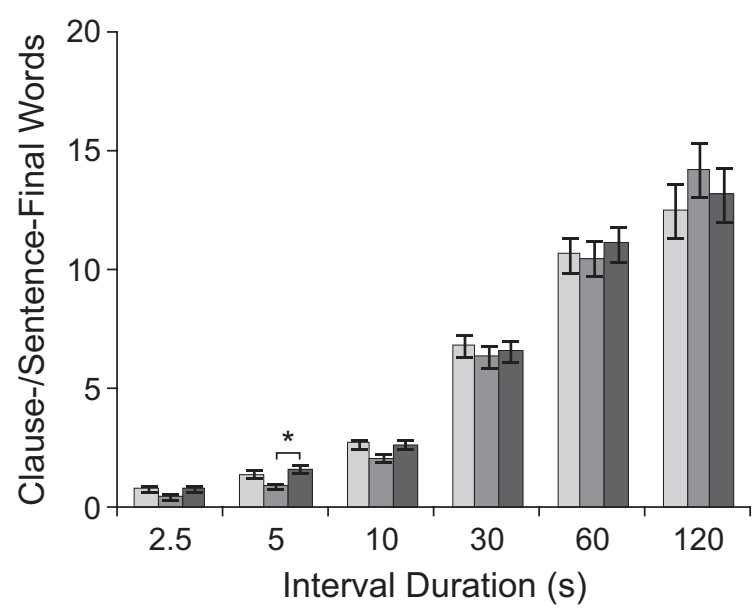

Fig. 3. Properties of fixated words during normal reading, self-caught mindless reading, and probe-caught mindless reading as a function of interval length. Means were calculated for (a) word length, (b) the natural logarithm of word frequency, and (c) the number of clause-/sentencefinal words. The error bars indicate standard errors of the mean, with significant differences indicated $(* p<.05)$.

Finally, two differences between self-caught and probecaught mind wandering in the present study further elucidate the possible role of meta-awareness in reading: Self-caught mind wandering was associated with longer fixations than was probecaught mind wandering, with the former also being associated with more off-text fixations immediately before mind-wandering reports than the latter. Because of the correlational nature of these data, it is not possible to determine whether the observed differences reflect increased cognitive demands of the dawning meta-awareness (which might draw resources away from reading) or whether pronounced deviations from normal reading behaviors instead serve as cues that the mind has wandered. This latter case raises the possibility of enhancing reading comprehension by sensitizing readers to aberrations in their gaze behaviors. For example, participants might be advised to keep an eye out (so to speak) for situations in which their eyes are moving especially slowly or not focusing on the text. Although future studies are necessary to test such predictions, our findings suggest that the mind and eye are tightly coupled, and that eye movements can be used to study even one of the most elusive aspects of the mind - self-reflection.

\section{Acknowledgments}

We thank Tomek Loboda, Simon Liversedge, Natasha Tokowicz, and two anonymous reviewers for their helpful comments on earlier drafts of this article.

\section{Declaration of Conflicting Interests}

The authors declared that they had no conflicts of interest with respect to their authorship or the publication of this article.

\section{Funding}

The work reported in this article was supported by the Institute of Educational Sciences (R305H030235). 

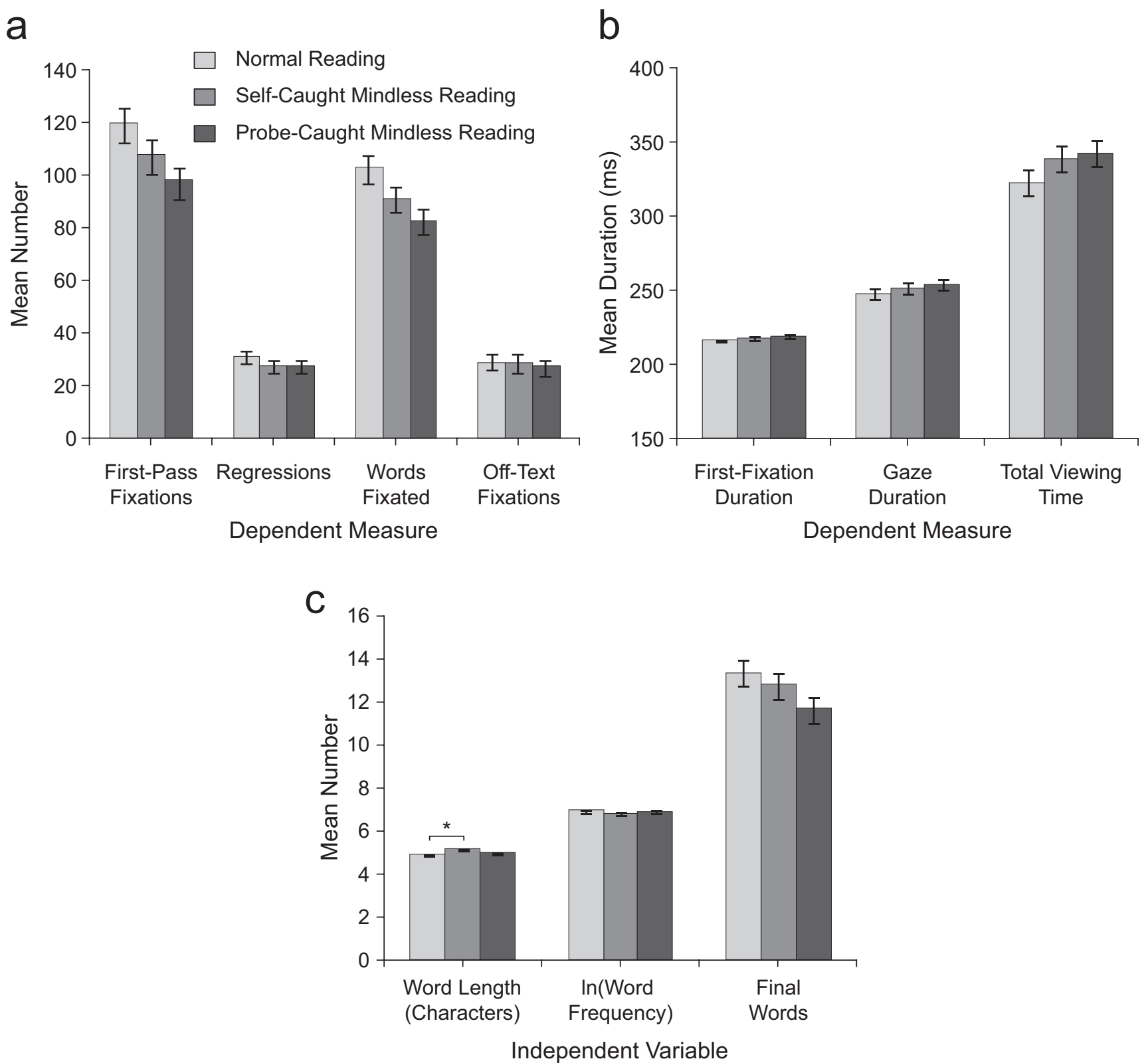

Fig. 4. Global eye movement fixation measures, fixation-duration measures, and word properties during the I20-s intervals after responses indicating normal reading, self-caught mindless reading, and probe-caught mindless reading. Means were calculated for (a) the number of first-pass fixations, interword regressions, words fixated, and off-text fixations; (b) first-fixation duration, gaze duration, and total viewing time; and (c) word length (in characters), natural logarithm of word frequency, and number of clause-/sentence-final words. The error bars indicate standard errors of the mean, with significant differences indicated $(* p<.05)$.

\section{Notes}

1. Although several experiments (Sayette et al., 2009; Schooler et al., 2004) indicate that mindless reading is amenable to study using selfpaced reading (in which participants press buttons to move through pages of text), pilot work suggested that the frequency of mind wandering observed using self-paced reading in conjunction with eye tracking is reduced because of the novelty of eye tracking and intrusions caused by recalibrating the eye tracker. We therefore ran 4 participants over several days (rather than many participants for one session each) to habituate them to the eye tracker and thereby offset the reduction in the expected number of mind-wandering episodes. Our efforts were successful because the observed number of episodes was only modestly reduced from the number observed in previous studies (e.g., see Schooler et al., 2004).

2. Because participants pressed buttons to respond to prompts and to self-report mind wandering, their eyes were often directed toward the keyboard. This made it impossible to examine differences in the rates of interword regressions immediately following self-caught versus probe-caught zone outs. 


\section{References}

Austen, J. (2008). Sense and sensibility. Retrieved from http://www .publicliterature.org/books/sense_and_sensibility (Original work published 1811)

Clifton, C., Staub, A., \& Rayner, K. (2007). Eye movements in reading words and sentences. In R.P.G. van Gompel, M.H. Fischer, W.S. Murray, \& R.L. Hill (Eds.), Eye movements: A window on mind and brain (pp. 341-371). New York: Elsevier.

Engbert, R., Nuthmann, A., Richter, E., \& Kliegl, R. (2005). SWIFT: A dynamical model of saccade generation during reading. Psychological Review, 112, 777-813.

Feng, G. (2006). Eye movements as time-series random variables: A stochastic model of eye movement control in reading. Cognitive Systems Research, 7, 70-95.

Francis, W.N., \& Kucera, H. (1982). Frequency analysis of English usage: Lexicon and grammar. Boston: Houghton Mifflin.

Inhoff, A.W., Eiter, B.M., \& Radach, R. (2005). Time course of linguistic information extraction from consecutive words during eye fixations in reading. Journal of Experimental Psychology: Human Perception and Performance, 31, 979-995.

Inhoff, A.W., \& Radach, R. (1998). Definition and computation of oculomotor measures in the study of cognitive processes. In G. Underwood (Ed.), Eye guidance in reading and scene perception (pp. 29-53). Amsterdam: Elsevier.

Just, M.A., \& Carpenter, P.A. (1980). A theory of reading: From eye fixations to comprehension. Psychological Review, 87, 329-354.

Kane, K.J., Brown, L.H., Little, J.C., Silvia, P.J., Myin-Germeys, I., $\&$ Kwapil, T.R. (2007). For whom the mind wanders, and when: An experience-sampling study of working memory and executive control in daily life. Psychological Science, 18, 614-621.

Kliegl, R., Grabner, E., Rolfs, M., \& Engbert, R. (2004). Length, frequency, and predictability effects of words on eye movements in reading. European Journal of Cognitive Psychology, 16, 262-284.

Liversedge, S.P., Paterson, K.B., \& Pickering, M.J. (1998). Eye movements and measures of reading time. In G. Underwood (Ed.), Eye guidance in reading and scene perception (pp. 55-75). Amsterdam: Elsevier.

Loboda, T.D. (2009). Pegasus [computer software]. (Available from the author, tomek.lobada@gmail.com)

McDonald, S.A., Carpenter, R.H.S., \& Shillcock, R.C. (2005). An anatomically-constrained, stochastic model of eye movement control in reading. Psychological Review, 112, 814-840.

Nuthmann, A., \& Engbert, R. (2009). Mindless reading revisited: An analysis based on the SWIFT model. Vision Research, 49, 322-336.

Pollatsek, A., Reichle, E.D., \& Rayner, K. (2006). Serial processing is consistent with the time course of linguistic information extraction from consecutive words during eye fixations in reading: A response to Inhoff, Eiter, and Radach (2005). Journal of Experimental Psychology: Human Perception and Performance, 32, 1485-1489.

Rayner, K. (1998). Eye movements in reading and information processing: 20 years of research. Psychological Bulletin, 124, 372-422.

Rayner, K. (2009). Eye movements in reading: Models and data. Journal of Eye Movement Research, 2, 1-10.
Rayner, K., \& Duffy, S.A. (1986). Lexical complexity and fixation times in reading: Effects of word frequency, verb complexity, and lexical ambiguity. Memory \& Cognition, 14, 191-201.

Rayner, K., \& Fischer, M.H. (1996). Mindless reading revisited: Eye movements during reading and scanning are different. Perception \& Psychophysics, 58, 734-747.

Rayner, K., Sereno, S.C., Morris, R.K., Schmauder, A.R., \& Clifton, C. (1989). Eye movements and on-line language comprehension processes. Language and Cognitive Processes, 4, 21-49.

Reichle, E.D., Liversedge, S.P., Pollatsek, A., \& Rayner, K. (2009). Encoding multiple words simultaneously in reading is implausible. Trends in Cognitive Sciences, 13, 115-119.

Reichle, E.D., Pollatsek, A., Fisher, D.L., \& Rayner, K. (1998). Toward a model of eye movement control in reading. Psychological Review, 105, 125-157.

Reichle, E.D., Rayner, K., \& Pollatsek, A. (2003). The E-Z Reader model of eye movement control in reading: Comparisons to other models. Behavioral \& Brain Sciences, 26, 445-476.

Reilly, R., \& Radach, R. (2006). Some empirical tests of an interactive activation model of eye movement control in reading. Cognitive Systems Research, 7, 34-55.

Salvucci, D.D. (2001). An integrated model of eye movements and visual encoding. Cognitive Systems Research, 1, 201-220.

Sayette, M.A., Reichle, E.D., \& Schooler, J.W. (2009). Lost in the sauce: The effects of alcohol on mind-wandering. Psychological Science, 6, 747-752.

Schilling, H.E.H., Rayner, K., \& Chumbley, J.I. (1998). Comparing naming, lexical decision, and eye fixation times: Word frequency effects and individual differences. Memory \& Cognition, 26, 1270-1281.

Schooler, J.W. (2002). Re-representing consciousness: Dissociations between consciousness and meta-consciousness. Trends in Cognitive Sciences, 6, 339-344.

Schooler, J.W., McSpadden, M., Reichle, E.D., \& Smallwood, J. (2010). Unnoticed nonsense: Mind-wandering can prevent people from realizing that they are reading gibberish. Manuscript submitted for publication.

Schooler, J.W., Reichle, E.D., \& Halpern, D.V. (2004). Zoning out during reading: Evidence for dissociations between experience and metaconsciousness. In D.T. Levin (Ed.), Thinking and seeing: Visual metacognition in adults and children (pp. 204-226). Cambridge, MA: MIT Press.

Smallwood, J., McSpadden, M., \& Schooler, J.W. (2008). When attention matters: The curious incident of the wandering mind. Memory \& Cognition, 36, 1144-1150.

Vitu, F., O’Regan, J.K., Inhoff, A.W., \& Topolski, R. (1995). Mindless reading: Eye-movement characteristics are similar in scanning letter strings and reading texts. Perception \& Psychophysics, 57, 352-364.

Warren, T., White, S.J., \& Reichle, E.D. (2009). Wrap up effects can be independent of interpretative processing: Evidence from eye movements. Cognition, 111, 132-137.

Yang, S.-N. (2006). An oculomotor-based model of eye movements in reading: The competition/interaction model. Cognitive Systems Research, 7, 56-69. 